The Astrophysical Journal, 552:L109-L112, 2001 May 10

(C) 2001. The American Astronomical Society. All rights reserved. Printed in U.S.A.

\title{
ULTRALUMINOUS X-RAY SOURCES IN EXTERNAL GALAXIES
}

\author{
A. R. King, ${ }^{1}$ M. B. Davies, ${ }^{1}$ M. J. Ward, ${ }^{1}$ G. Fabbiano, ${ }^{2}$ and M. Elvis ${ }^{2}$ \\ Received 2001 February 22; accepted 2001 April 4; published 2001 April 30
}

\begin{abstract}
We investigate models for the class of ultraluminous nonnuclear X-ray sources (i.e., ultraluminous compact X-ray sources [ULXs]) seen in a number of galaxies and probably associated with star-forming regions. Models in which the X-ray emission is assumed to be isotropic run into several difficulties. In particular, the formation of sufficient numbers of the required ultramassive black hole X-ray binaries is problematic, and the likely transient behavior of the resulting systems is not in good accord with observation. The assumption of mild X-ray beaming suggests instead that ULXs may represent a short-lived but extremely common stage in the evolution of a wide class of X-ray binaries. The best candidate for this is the phase of thermal-timescale mass transfer that is inevitable in many intermediate- and high-mass X-ray binaries. This in turn suggests a link with the Galactic microquasars. The short lifetimes of high-mass X-ray binaries would explain the association of ULXs with episodes of star formation. These considerations still allow the possibility that individual ULXs may contain extremely massive black holes.
\end{abstract}

Subject headings: accretion, accretion disks — binaries: close — X-rays: stars

\section{INTRODUCTION}

The existence in spiral galaxies of off-nuclear X-ray sources whose luminosities appear significantly larger than the Eddington limit for a $1 M_{\odot}$ object has been known for some time (Fabbiano 1989). These sources are distinct from the weak active galactic nuclei (AGNs) present in many spiral galaxies known as LINERs (Ho et al. 1997), although in at least one case (M33; see Dubus et al. 1997) they may be confused with AGNs. Recently, considerable effort has been devoted to interpreting the properties of these ultraluminous compact X-ray sources (ULXs; e.g., Makishima et al. 2000 and references therein). A key to understanding their nature may be that they appear to occur preferentially, although not exclusively, in regions of star formation (Zezas, Georgantopoulos, \& Ward 1999; Roberts \& Warwick 2000; Fabbiano, Zezas, \& Murray 2001). In this Letter, we investigate models for the ULXs.

Bright, nonnuclear X-ray point sources in galaxies are divided into two groups: (1) accreting neutron stars and black holes and (2) young supernova remnants. The luminosities of the first group, but not the second, are constrained by the Eddington limit:

$$
L_{\mathrm{X}} \lesssim L_{\mathrm{Edd}} \simeq \frac{4 \pi G M_{1} m_{p} c}{\sigma_{\mathrm{T}}} \simeq 1.3 \times 10^{38} m_{1} \mathrm{ergs} \mathrm{s}^{-1},
$$

where $\sigma_{\mathrm{T}}$ is the Thomson cross section and $m_{1}$ is the accretor mass $M_{1}$ in units of solar mass. This constraint applies to any nonexplosive source, whether powered by accretion or other means such as nuclear burning.

Evasions of the limit are possible but rare. In the transient system A0538-66, a magnetic neutron star accretes from the wind of a Be-star companion. The system sometimes has superEddington luminosities $L_{\mathrm{X}} \simeq 10^{39} \mathrm{ergs} \mathrm{s}^{-1}$ (White \& Carpenter 1978), but these may result from the reduction in the electron-

\footnotetext{
' Department of Physics and Astronomy, University of Leicester, University Road, Leicester, LE1 7RH, UK; ark@ star.le.ac.uk.

${ }^{2}$ Harvard-Smithsonian Center for Astrophysics, 60 Garden Street, Cambridge, MA 02138 .
}

scattering cross section below $\sigma_{\mathrm{T}}$ in the $\sim 10^{11} \mathrm{G}$ magnetic field of the neutron star pervading the accretion columns. Thus, if we regard the observed variability of ULXs as ruling out the identification as supernova remnants, a straightforward interpretation as nonexplosive sources requires black holes with masses $M_{1} \gtrsim 50-100 M_{\odot}$, accreting at rates that on occasion can exceed $\sim 10^{-6} M_{\odot} \mathrm{yr}^{-1}$. As we shall see, while individual ULXs could harbor such masses, there are major difficulties with such a picture as an explanation for the ULXs as a class. Accordingly, we consider models in which the X-ray emission is assumed to be significantly beamed. In this case, ULXs may correspond to a relatively short-lived but common epoch of the evolution of close intermediate- or high-mass X-ray binaries, perhaps the thermal-timescale mass transfer phase following the normal X-ray phase.

\section{LUMINOSITIES, LIFETIMES, MASSES, AND BIRTHRATES}

We first consider the restrictions placed by observation on accretion models for the ULXs. We assume that a compact object of mass $M_{1}$ accretes from a mass reservoir (e.g., a companion star) of mass $M_{2}$. We denote the mean observed number of ULXs per galaxy as $n$, the beaming factor as $b(=\Omega / 4 \pi$, where $\Omega$ is the solid angle of emission), the duty cycle (which equals the time that the source is active as a fraction of its lifetime) as $d$, and define an "acceptance rate" $a$ as the ratio of mass accreted by $M_{1}$ to that lost by $M_{2}$, i.e., the mean accretion rate $\dot{M}_{1}=a\left(-\dot{M}_{2}\right)$. We further define $L_{\mathrm{sph}}$ as the apparent X-ray (assumed bolometric) luminosity of a source, given by the assumption of isotropic (spherical) emission, and let $L_{40}=L_{\mathrm{sph}} / 10^{40} \mathrm{ergs} \mathrm{\textrm {s } ^ { - 1 }}$. From these definitions, it follows that the luminosity

$$
L=b L_{\mathrm{sph}}=10^{40} b L_{40} \operatorname{ergs~\mathrm {s}^{-1}}
$$

and the minimum accretor mass, if the source is not to exceed 
the Eddington limit, is

$$
M_{1} \gtrsim 10^{2} b L_{40} M_{\odot}
$$

The total number of such sources per galaxy is

$$
N=\frac{n}{b d}
$$

with a minimum mean accretion rate during active phases of

$$
\dot{M}_{\text {active }}=\frac{\dot{M}_{1}}{d}=-\frac{\dot{M}_{2} a}{d}>10^{-6} b L_{40} M_{\odot} \mathrm{yr}^{-1} .
$$

The mass-loss rate from $M_{2}$ is thus

$$
-\dot{M}_{2}>10^{-6} \frac{b d}{a} L_{40} M_{\odot} \mathrm{yr}^{-1}
$$

and the lifetime of a source is

$$
\tau=-\frac{M_{2}}{\dot{M}_{2}} \lesssim 10^{6} \frac{m_{2} a}{b d L_{40}} \mathrm{yr}
$$

with $m_{2}=M_{2} / M_{\odot}$, leading to a required birthrate per galaxy of

$$
B=\frac{N}{\tau} \gtrsim \frac{n}{b d} \frac{b d L_{40}}{10^{6} m_{2} a}=10^{-6} \frac{n L_{40}}{m_{2} a} \mathrm{yr}^{-1}
$$

It is important to note here that the required birthrate is independent of beaming (and the duty cycle): the greater intrinsic source population $N$ required by beamed sources with $b d<1$ (see eq. [4]) is compensated by their longer lifetimes (see eq. [7]).

\section{UNBEAMED MODELS}

For an unbeamed model, we set $b=1$ and recover from equation (3) the requirement that $M_{1} \gtrsim 10^{2} L_{40}$. We note immediately that some ingenuity is required (see Makishima et al. 2000) to make these masses compatible with the characteristic observed X-ray temperatures of 1-2 keV, whereas these are natural values for the lower masses that we shall find below for beamed models. With $b=1$, equation (5) gives $\dot{M}_{\text {active }} \gtrsim$ $10^{-6} L_{40} M_{\odot} \mathrm{yr}^{-1}$. This effectively forces the mass reservoir to be a companion star; except for extremely high black hole masses $M_{1} \gtrsim 3 \times 10^{4} M_{\odot}$, Bondi accretion from even a relatively dense part of the interstellar medium (ISM) is inadequate, giving a rate of

$$
\dot{M}_{\text {Bondi }} \simeq 1 \times 10^{-11} \frac{m_{100}^{2}\left(\rho / 10^{-24} \mathrm{~g} \mathrm{~cm}^{-3}\right)}{u_{10}^{2}+c_{10}^{2}} M_{\odot} \mathrm{yr}^{-1},
$$

where $m_{100}=M_{1} / 100 M_{\odot}, \rho$ is the mass density of the ambient ISM, and $u_{10}$ and $c_{10}$ are the relative speed of the hole and local ISM and the local ISM sound speed, respectively, both in units of $10 \mathrm{~km} \mathrm{~s}^{-1}$. While individual ULXs might contain black holes of masses $M_{1} \gtrsim 3 \times 10^{4} M_{\odot}$, it seems improbable that galaxies like the Antennae should contain about 10 accreting examples. Unbeamed models for the ULX class thus have to invoke a class of extremely massive X-ray binaries. As we shall see, this may be a potential problem. Accepting that suitable binaries could in principle form, there are the usual constraints familiar from low-mass X-ray binary evolution (see Kalogera \& Webbink 1996). Particularly important are that (1) the binary must be wide enough so that the progenitor of the compact star (here an $\sim 100 M_{\odot}$ black hole) is able to fit inside its Roche lobe (otherwise it will provoke commonenvelope evolution) and (2) the binary must be able to provide the inferred minimum accretion rate $\dot{M}_{1} \sim 10^{-6} M_{\odot} \mathrm{yr}^{-1}$. The first constraint immediately sets a scale since main-sequence stars of masses $\gtrsim 100 M_{\odot}$ have radii $\gtrsim 10^{3} R_{\odot}$ (e.g., Stothers \& Chin 1999). Using Kepler's law and assuming $M_{1} \gg M_{2}$, this implies binary periods

$$
P \gtrsim 1 m_{100}^{-0.5} \mathrm{yr} \text {. }
$$

We can compare this with the critical period beyond which the accretion disk around the black hole cannot be thermally stable, and the system must therefore be transient. From King (2000), we find that

$$
P_{\text {crit }} \sim 4 m_{100}^{1 / 8} m_{2}^{1 / 8} \text { days. }
$$

We see that unbeamed ULXs must be transient. Hence, the inferred $\dot{M}_{1} \sim 10^{-6} M_{\odot} \mathrm{yr}^{-1}$ now refers to the outburst state only: this is advantageous since persistent mass transfer rates $-\dot{M}_{2}$ of this order would have been difficult to explain. To fill the Roche lobe in a binary with the period given by equation (10) requires an extended star (note that the binary period essentially fixes the mean density of this star uniquely; see, e.g., Frank, King, \& Raine 1992). From the formulae of King (1988; see Ritter 1999), we see that an evolved star with a helium core mass $M_{c} \sim$ 0.4-0.5 $M_{\odot}$ will fill the Roche lobe, independently of the total donor mass $M_{2}>M_{c}$. Mass transfer is driven by the nuclear expansion of the star, at the rate of

$$
-\dot{M}_{2} \simeq 1 \times 10^{-7} P_{\mathrm{yr}}^{0.93} m_{2}^{1.47} M_{\odot} \mathrm{yr}^{-1}
$$

where $P_{\mathrm{yr}}$ is the binary period in years (see eq. [7] of King, Kolb, \& Burderi 1996). Thus, even a duty cycle $d$ as long as $10 \%$ would yield mean accretion rates of the required order; i.e., $\dot{M}_{1}=\dot{M}_{\text {active }}=\dot{M}_{1} d^{-1} \sim 10^{-6} M_{\odot} \mathrm{yr}^{-1}$ during outbursts.

However, applying the simple irradiated-disk theory of King \& Ritter (1998), we predict that the accretion rate $\dot{M}_{\text {active }}$ should decay linearly from its initial peak down to zero on a 10-20 yr timescale. This is not easily compatible with a comparison of ROSAT and Chandra data. This may not be a crucial objection to this type of unbeamed model for ULXs; the theory of outbursts in large irradiated disks is complicated, even without adding further difficulties such as radiation-induced disk warping.

A more serious objection to unbeamed models is the one touched on above, namely, that they require an $\sim 100 M_{\odot}$ black hole to coexist in a binary with an evolved companion star. Mass loss from very massive stars with nonzero metallicity is usually thought to be so strong that the final black hole mass is much smaller than the initial stellar mass (see Baraffe, Heger, \& Woosley 2001 for a recent view and Papaloizou 1973 for a possible objection). Assuming coeval formation of the two stars in the binary rules out a primordial origin for the black hole progenitor and would thus require a progenitor with a mass $\gg 100 M_{\odot}$. If the initial mass function within the stellar clusters is close to that of Salpeter, we conclude that the number of stars formed having masses $\geq 100 M_{\odot}$ is a factor of $\sim 100$ lower 
than the number of stars having initial masses in the range $10 M_{\odot} \leq m \leq 100 M_{\odot}$. We would therefore expect X-ray binaries containing neutron stars and lower mass black holes to outnumber markedly those containing higher mass black holes. The X-ray luminosities of the systems observed in the Antennae (Fabbiano et al. 2001) contradict this, assuming that all systems are unbeamed, although the number of low-luminosity systems is not currently well known.

Alternatively, an $\sim 100 M_{\odot}$ black hole may have only recently gained a new stellar partner. Such black holes may be produced within dense clusters through the merger of lower mass black holes (see, e.g., Lee 1993, 1995). Indeed, this has been proposed as the origin of the moderate-mass black hole inferred to be present in the central regions of M82 (Matsushita et al. 2000; Matsumoto et al. 2001; Kaaret et al. 2001). Although it is possible that a moderate-mass black hole produced in a central cluster of M82 has gained a stellar companion by some dynamical process (tidal capture or via an exchange encounter involving a binary), this scenario is unlikely to work for the systems observed in the Antennae, where the ULXs are observed to be strongly associated with the young star clusters that are located some distance from the galactic nuclei (Fabbiano et al. 2001). Any massive black hole would therefore have to be formed within these stellar clusters and not within the nuclear clusters. To produce moderate-mass black holes within a cluster via the successive merger of lower mass objects, the potential well of the cluster has to be sufficiently deep to retain the black holes. This can be the case for a stellar cluster in the nucleus of a galaxy but is not true for globular clusters in which the typical escape speed is far too low to retain black hole binaries as they are hardened via encounters. This has been suggested as the reason for the absence of black hole binary systems in globular clusters (Sigurdsson \& Hernquist 1993; Kulkarni, Hut, \& McMillan 1993).

A population of $\sim 100 M_{\odot}$ black holes originating from a much earlier generation of effectively zero-metallicity stars seems unlikely to explain the ULXs observed in the Antennae (Fabbiano et al. 2001) since these black holes would be distributed throughout the galactic halo, and so the probability of picking up stars from the young stellar clusters via dynamical encounters within the last $\sim 10^{7} \mathrm{yr}$ is extremely low.

\section{BEAMED MODELS}

Since unbeamed models run into difficulties because of the required black hole mass of $\sim 100 M_{\odot}$ and the need for a companion, we consider the effect of assuming that the observed Xrays are mildly beamed. The simplest candidate mechanism is the idea that the accretion disk around an accreting black hole has a much lower scattering optical depth over a restricted range of solid angles (e.g., the rotational poles) than in other directions. Almost all the emitted X-rays would therefore emerge in these directions. A beaming factor $b \leq 0.1$ would bring the required minimum accretor mass (see eq. [3]) into the range of $M_{1} \lesssim$ $10 M_{\odot}$ commonly found in dynamical measurements of X-ray binaries, particularly quiescent soft X-ray transients (e.g., Charles 1998), while $b \lesssim 0.01$ would bring $M_{1}$ down to neutron star values. In addition, this kind of disk geometry, i.e., a thick disk with a central funnel, may actually radiate a total luminosity in excess of the Eddington limit (Jaroszynski, Abramowicz, \& Paczyński 1980; Abramowicz, Calvani, \& Nobili 1980). Thus, such modest $b$-values may allow quite large apparent luminosities for perfectly standard black hole or neutron star masses. The obvious implication is that beamed ULXs might represent some short-lived phase in the evolution of a large class of $\mathrm{X}$-ray binaries; from equation (7), we find that $\tau \lesssim$ $10^{7} m_{2} a /(b / 0.1) d$ yr.

A good candidate for such a phase is an episode of thermaltimescale mass transfer. These are extremely common, occurring when the donor has a radiative envelope and either $(a)$ is somewhat more massive than the accretor and/or $(b)$ first fills its Roche lobe as it expands across the Hertzsprung gap. In general, both cases give rise to highly super-Eddington mass transfer rates. Case $a$ is unavoidable, for example, in any neutron star binary with an intermediate mass ( 2-4 $M_{\odot}$ donor); King \& Ritter (1999) and Podsiadlowski \& Rappaport (2000) show that Cygnus X-2 is a survivor of such an episode, in which $-\dot{M}_{2}$ reached values of order $\sim 10^{-6} M_{\odot} \mathrm{yr}^{-1}$ and the excess mass transfer is simply blown away from the system rather than resulting in common-envelope evolution (see also King \& Begelman 1999 and Kolb et al. 2000). Case $b$ requires only a reasonably wide binary separation after formation of the compact star and clearly benefits from a large initial phase space. Either case $a$ or case $b$ is also the likely path for all high-mass X-ray binaries such as Cyg X-1 once the current wind-fed X-ray phase ends.

Until recently, it has generally been assumed that thermaltimescale episodes are unobservable because they are short and that, without beaming, X-rays could not emerge from the superEddington accretion flow at all. We investigate here the possibility that ULXs could be systems in this phase, where beaming allows us to see the X-rays.

The thermal-timescale mass transfer rate from a donor near the upper main sequence is roughly (see King \& Begelman 1999)

$$
-\dot{m}_{2} \simeq 3 \times 10^{-8} m_{2}^{2.6} M_{\odot} \mathrm{yr}^{-1}
$$

Comparing with the Eddington accretion rate, we can calculate an acceptance rate of

$$
a=0.43 m_{1} m_{2}^{-2.6}
$$

and thus a lifetime (assuming $d=1$ )

$$
\tau \lesssim 4.3 \times 10^{6}(b / 0.1)^{-1} L_{40}^{-1} m_{1} m_{2}^{-1.6} \mathrm{yr}
$$

and birthrate

$$
B \gtrsim 2.3 \times 10^{-6} n(b / 0.1) L_{40} m_{1}^{-1} m_{2}^{1.6} \mathrm{yr}^{-1}
$$

per galaxy. In particular, for a system like Cyg X-2, which has $m_{1} \simeq 1.4 M_{\odot}$ and $m_{2} \simeq 3 M_{\odot}$ (King \& Ritter 1999; Podsiadlowski \& Rappaport 2000; Kolb et al. 2000), we get a required birthrate

$$
B \gtrsim 1 \times 10^{-6} n(b / 0.1) L_{40} \mathrm{yr}^{-1}
$$

We may compare this with the Galactic birthrate of $\sim 10^{-6}$ to $10^{-7} \mathrm{yr}^{-1}$ deduced for Cyg X-2-like systems (King \& Ritter 1999; Podsiadlowski \& Rappaport 2000; Kolb et al. 2000). For a high-mass black hole system like Cyg X-1, both $m_{1}$ and $m_{2}$ are probably significantly higher, raising $B$ by as much as an order of magnitude. However, the short X-ray lifetime of $\sim 10^{5} \mathrm{yr}$ of this X-ray phase requires a correspondingly high Galactic birthrate of approximately a few times $10^{-5} \mathrm{yr}^{-1}$, again allowing a significant ULX population. X-ray binaries reach 
the thermal-timescale phase in a timescale comparable with the main-sequence lifetime of the donor. Thus, ULXs descending from high-mass X-ray binaries would naturally be associated with a young stellar population, as required by observation.

A possible example of a ULX in the Galaxy is GRS $1915+105$, where $L_{\mathrm{X}} \sim 1 \times 10^{39} \mathrm{ergs} \mathrm{s}^{-1}$ (e.g., Belloni et al. 1997). Since this is a microquasar, with a radio jet axis at about $70^{\circ}$ to the line of sight (see Mirabel \& Rodríguez 1999), only mild beaming $(b \sim 0.6)$ is possible, even assuming that we view the system at the edge of the X-ray beam. However, this is indeed sufficient to reduce the luminosity to sub-Eddington values. Moreover, such a geometrical alignment is quite reasonable since it offers an explanation for the very unusual longterm behavior of GRS $1915+105$. The system was not detected in X-rays until 1992, and since then it has remained persistently bright with only short interruptions. The usual explanation that this is an accretion disk instability, prolonged by self-irradiation by X-rays (see King \& Ritter 1998), would require an implausibly large disk mass. An attractive alternative is that the $\mathrm{X}$-ray light curve reflects slight changes in the X-ray beaming, which would have decreased enough in 1992 to allow us to see the X-rays.

\section{CONCLUSIONS}

We have considered models for the ULX class and reached the following conclusions:

1. Unbeamed models probably require a black hole of $M_{1} \gtrsim 100 M_{\odot}$ in an $\sim 1$ yr binary orbit with an evolved donor star. Forming such a system presents considerable difficulties, and even then the likely transient behavior of the accretion disk in such a wide system is hard to reconcile with observation. It is still possible that an individual ULX may contain a very massive black hole $\left(M_{1} \gtrsim 3 \times 10^{4} M_{\odot}\right)$, perhaps accreting from the ISM.

2. The assumption of mild beaming $(b \sim 0.1-0.01)$ reduces $M_{1}$ to values already observed for Galactic X-ray binaries and suggests that ULXs represent a short-lived phase of their evo- lution. The most likely candidate for this is the thermal-timescale mass transfer episode that is inevitable in a very wide class of intermediate- and high-mass X-ray binaries. This in turn suggests a link to the Galactic microquasars (see the remarks by A. R. King quoted in Mirabel \& Rodríguez 1999). The short donor lifetime in high-mass X-ray binaries would explain why ULXs are associated with young stellar populations.

The major theoretical uncertainty for the second conclusion is whether or not beaming is a natural consequence of high accretion rates. Only large numerical simulations can address this question. For this type of model, it is perhaps encouraging to know that not only are the X-ray spectra fairly similar to those of Galactic black hole systems but that Kubota et al. (2001) observed X-ray spectral transitions typical of such a source in two ULXs. The same type of spectral and timing variability has also been seen in the X-9 source in the M81 field (La Parola et al. 2001). There are several possible observational tests of these ideas. First, continued X-ray monitoring with a view to detecting possible changes in beaming geometry is clearly worthwhile. We note, however, that X-ray eclipses are unlikely in any beamed model, assuming that the $\mathrm{X}$-ray beam axis is normal to the binary plane. Optical identifications of ULXs might allow at least two kinds of test: If the total X-ray luminosities really are as large as predicted if there is no beaming, one might expect to detect photoionization nebulae around ULXs. If, on the other hand, ULXs are beamed, and thus of normal stellar mass, one might hope ultimately to detect a spectroscopic period (say tens of days) in a ULX within the Local Group.

We thank Mike Garcia, Jim Pringle, Hans Ritter, Tim Roberts, Rashid Sunyaev, and Pete Wheatley for discussions. M. B. D. gratefully acknowledges the support of a University Research Fellowship from the Royal Society. Theoretical astrophysics research at Leicester is supported by a PPARC rolling grant. This work was supported in part by NASA contract NAS8-39073 (CXC).

\section{REFERENCES}

Abramowicz, M. A., Calvani, M., \& Nobili, L. 1980, ApJ, 242, 772

Baraffe, I., Heger, A., \& Woosley, S. E. 2001, ApJ, 550, 890

Belloni, T., Méndez, M., King, A. R., van der Klis, M., \& van Paradijs, J. 1997, ApJ, 488, L109

Charles, P. 1998, in Theory of Black Hole Accretion Disks, ed. M. A. Abramowicz, G. Bjornsson, \& J. E. Pringle (Cambridge: Cambridge Univ. Press), 1

Dubus, G., Charles, P. A., Long, K., \& Hakala, P. J. 1997, ApJ, 490, L47

Fabbiano, G. 1989, ARA\&A, 27, 87

Fabbiano, G., Zezas, A., \& Murray, S. S. 2001, ApJ, in press

Frank, J., King, A. R., \& Raine, D. J. 1992, Accretion Power in Astrophysics (2d ed.; Cambridge: Cambridge Univ. Press), chap. 4

Ho, L., Filippenko, A. V., Sargent, W. L., \& Peng, C. Y. 1997, ApJS, 112, 391

Jaroszynski, M., Abramowicz, M. A., \& Paczyński, B. 1980, Acta Astron., 30,1

Kaaret, P., Prestwich, A. H., Zezas, A., Murray, S. S., Kim, D.-W., Kilgard, R. E., Schlegel, E. M., \& Ward, M. J. 2001, MNRAS, 321, L29

Kalogera, V., \& Webbink, R. F. 1996, ApJ, 458, 301

King, A. R. 1988, QJRAS, 29, 1 2000, MNRAS, 315, L33

King, A. R., \& Begelman, M. C. 1999, ApJ, 519, L169

King, A. R., Kolb, U., \& Burderi, L. 1996, ApJ, 464, L127

King, A. R., \& Ritter, H. 1998, MNRAS, 293, L42
King, A. R., \& Ritter, H. 1999, MNRAS, 309, 253

Kolb, U., Davies, M. B., King, A. R., \& Ritter, H. 2000, MNRAS, 317, 438 Kubota, A., Mizuno, T., Makishima, K., Fukazawa, Y., Kotoku, J., Ohnishi, T., \& Tashiro, M. 2001, ApJ, 547, L119

Kulkarni, S. R., Hut, P., \& McMillan, S. 1993, Nature, 364, 421

La Parola, V., Peres, G., Fabbiano, G., Kim, D.-W., \& Bocchino, F. 2001, ApJ, in press

Lee, H. M. 1995, MNRAS, 272, 605

Lee, M. H. 1993, ApJ, 418, 147

Makishima, K., et al. 2000, ApJ, 535, 632

Matsumoto, H., Tsuru, T. G., Koyama, K., Awaki, H., Canizares, C. R., Kawai, N., Matsushita, S., \& Kawabe, R. 2001, ApJ, 547, L25

Matsushita, S., Kawabe, R., Matsumoto, H., Tsuru, T., Kohno, K., Morita, K., Okumura, S. K., \& Vila-Vilaro, B. 2000, ApJ, 545, 107

Mirabel, I. F., \& Rodríguez, L. F. 1999, ARA\&A, 37, 409

Papaloizou, J. C. B. 1973, MNRAS, 162, 143

Podsiadlowski, Ph., \& Rappaport, S. A. 2000, ApJ, 529, 946

Ritter, H. 1999, MNRAS, 309, 360

Roberts, T., \& Warwick, R. 2000, MNRAS, 315, 98

Sigurdsson, S., \& Hernquist, L. 1993, Nature, 364, 423

Stothers, R. B., \& Chin, C. W. 1999, ApJ, 522, 960

White, N. E., \& Carpenter, G. F. 1978, MNRAS, 183, 11P

Zezas, A., Georgantopoulos, I., \& Ward, M. J. 1999, MNRAS, 308, 302 